Original Article

\title{
The influences of the mental practice on the quality of life of the stroke patients
}

\author{
JuHyung PARK, MSc, OT ${ }^{1)}$ \\ 1) Department of Occupational Therapy, Health Science College, Cheongju University: \\ 298 Daesung-ro, Cheongwon-gu, Cheongju 363-764, Republic of Korea
}

\begin{abstract}
Purpose] The purpose of this research is to take a look at the influences of the mental practice on the quality of life of stroke patients. [Subjects and Methods] Through a randomization of 32 stroke patients, this research allocated the experimental group $(n=15)$ and the control group $(n=17)$. Regarding the experimental group, the imagination practice intervention and the ordinary rehabilitation therapy had been applied 1 time a day and 5 times a week for 4 weeks. Regarding the control group, the ordinary rehabilitation therapy had been provided during the same session as that of the experimental group. In order to measure the quality of life, the SS-QOL was used. [Results] The mental practice and CON groups showed significant differences with the SS-QOL scores. [Conclusion] Through the result of this research, we were able to find out that the mental practice intervention is effective with regard to the improvement of the quality of life of the stroke patients. Regarding the researches that will take place later on, the researches which achieved the securing of even more experiment subjects and the control of the diverse variables of the quality of life will be needed.

Key words: Mental practice, Quality of life, Stroke
\end{abstract}

(This article was submitted Apr. 4, 2017, and was accepted Jun. 12, 2017)

\section{INTRODUCTION}

The mental practice is a method of intervention which can make the subjects learn the exercise skills and improve the ability to engage in an exercise by having the subjects imagine the movements in their minds and carry them out without actually moving the body and which can improve the ability to carry out the exercises ${ }^{1)}$. Recently, as a method for the learning and improvement of the exercise skills, the imagination practice interventions have been used in the diverse therapy and rehabilitation areas ${ }^{2)}$, and a strong point of this intervention is that the training is possible without any expensive treatment tools without regard to the time and the place ${ }^{3)}$. If we take a look at the researches that applied the mental practice with the stroke patients as the subjects, Korn said that he carried out an exercise mental practice for the improvement of the exercise execution capabilities with the stroke patients as the subjects and that the balancing ability while sitting down of the group which carried out the mental practice significantly improved more than that of the group which carried out the rehabilitation therapy ${ }^{4}$. According to his research results, Park reported the improvement of the manual function and the capability of carrying out the everyday lives through the positive changes of the muscular activities of the stroke patients ${ }^{5)}$.

Generally, the quality of life of the stroke patients has been emphasized very much in the field of rehabilitation ${ }^{6}$. And it has been known that such a quality of life has the significant correlation with the functions and the ability to carry on the everyday lives of the patients themselves ${ }^{7}$. If we take a look at the results of the researches related to the imagination training that were reported previously, in most of the researches, there have been the limitations to the improvements and others of the like of the balance, the manual function, and the ability to carry out the everyday lives. As such, the results of the researches on the influences of the mental practice due to this on the quality of life of the stroke patients are on an insignificant level. As 
Table 1. General characteristic of the subjects

\begin{tabular}{lcc}
\hline & Mental practice & CON \\
\hline Gender (Male/Female) & $10 / 5$ & $11 / 6$ \\
Age (years) & $62 \pm 5.9$ & $67 \pm 7.2$ \\
Onset (month) & $13 \pm 8.9$ & $22 \pm 19.2$ \\
Paretic side (Right/Left) & $8 / 7$ & $10 / 7$ \\
\hline All variables are mean \pm standard deviation (SD). CON: \\
control group
\end{tabular}

such, in this research, it is intended to take a look at the influences of the mental practice on the quality of life of the stroke patients.

\section{SUBJECTS AND METHODS}

The subjects of this research were the 32 patients who received the diagnosis of the hemiplegia due to stroke and had been receiving the hospitalized therapy at Hospital D in Korea. The subjects received an explanation about the purpose and methods of the study prior to their participation and provided informed consent according to the ethical principles of the Declaration of Helsinki. The criteria for the selection of the subjects of this research are as below. The persons who had the laterality hemiplegia for over 6 months. The persons whose scores are 24 points or higher in the Korean version of the Mini-Mental State Examination (MMSE-K). The persons whose averages are 2.26 or below in the Vividness of Movement Imagery Questionnaire (VMIQ). The persons who do not have any problems in communicating. The persons who do not have hemi-neglect. The persons who do not have any hearing and visual impairments. As a person who shows the functions of the pronation of the wrist with the manual function, the extending of the wrist by at least 20 in the condition of bending the wrist, and the extending of the metacarpophalangeal joint to at least 10, a person who can grasp and place the things with the affected side. The general characteristics of the subjects are noted in Table 1.

By randomizing the 32 stroke patients who participated in the experiment, we proceeded by dividing them into the experimental group and the control group. The experimental group had received the traditional, occupational therapy for 20 minutes a day, 5 times a week, and for 4 weeks. And the imagination practices for 10 minutes each were carried out at the end of the occupational therapy time. And the total time of the imagination training that was applied was 200 minutes. In contrast to this, regarding the control group, the traditional, occupational therapy and the physical therapy had been carried out for 30 minutes a day, for 5 times a week, and for 4 weeks. The mental practice was carried out in the occupational therapy room with the quiet environment and when the subjects were in the condition in which their physical and psychological conditions were stable. Regarding all the training programs, the files that were recorded in advance with the length of 10 minutes were used and applied. The programs that were used for the training were a total of three, including turning the pages of a book, putting in the beans, and piling up the plastic cups.

The experimental design in this study was the pre-test and the post-test with the control group design for the 4 weeks intervention. The pre-test and post-test measurements were obtained 1 day before and after the training, respectively, using the clinical measures. The evaluations of the quality of life of the subjects were measured by using the Stroke-Specific Quality of Lives (SS-QOL) for both before and after the intervention for both groups. The 12 domains of the SS-QOL were the energy, the role in the family, the usage of language, the moving, the mood, the individual's personality, the self-help activities, the social role, the ability to think, the manual function, the eyesight, and the occupational production activities. The number of the questions was a total of 49 questions. And it is an evaluation tool with the 5-point scale. According to a report on a research with the stroke patients in Korea as the subjects, the reliability appeared high at 0.806). The SPSS ver. 18.0 was used to calculate the means and the standard deviations. The descriptive statistics were used to analyze the general characteristics of the subjects. The differences with the quality of life scores between the 2 groups were investigated using the independent t-test. For all the data, the significance was accepted at the values of $\mathrm{p}<0.05$.

\section{RESULTS}

Table 2 shows the quality of life scores before and after the mental practice. The mental practice and the CON groups showed the significant differences in the following measures: SS-QOL's $(\mathrm{p}<0.05)$.

\section{DISCUSSION}

In this research, it was intended to find out the possibility of the therapeutic effects of the mental practice with regard to the quality of life of the patient aspect by applying the mental practice to the stroke patients who were the subjects. As a result, the significant therapeutic improvements of the quality of life of the stroke patients who received the mental practice interventions were observed. If we take a look by detailed item, in most of the items, no significant result could be obtained. 
Table 2. Comparison of quality of lifes of each group

\begin{tabular}{|c|c|c|c|c|}
\hline & \multicolumn{2}{|c|}{ mental practice } & \multicolumn{2}{|c|}{$\mathrm{CON}$} \\
\hline & Pre-test & Post-test & Pre-test & Post-test \\
\hline SS-QOL* & $152.72 \pm 19.3$ & $164.28 \pm 14.24$ & $153.26 \pm 15.79$ & $154.59 \pm 16.64$ \\
\hline Energy & $9.22 \pm 2.98$ & $10.29 \pm 3.56$ & $9.51 \pm 2.89$ & $10.29 \pm 2.91$ \\
\hline Family roles & $9.22 \pm 2.98$ & $10.84 \pm 2.79$ & $9.51 \pm 2.89$ & $9.10 \pm 3.67$ \\
\hline Language & $20.67 \pm 5.36$ & $20.41 \pm 3.03$ & $20.25 \pm 5.33$ & $20.98 \pm 4.29$ \\
\hline Mobility & $14.71 \pm 5.07$ & $15.51 \pm 5.04$ & $14.05 \pm 4.73$ & $14.91 \pm 5.67$ \\
\hline Mood & $17.69 \pm 4.39$ & $20.23 \pm 5.21$ & $17.95 \pm 4.03$ & $18.21 \pm 3.19$ \\
\hline Personality & $14.76 \pm 5.18$ & $14.28 \pm 4.37$ & $15.31 \pm 5.35$ & $15.71 \pm 5.23$ \\
\hline Self care* & $13.22 \pm 4.13$ & $16.29 \pm 4.57$ & $13.75 \pm 4.75$ & $12.86 \pm 4.85$ \\
\hline Social roles & $11.00 \pm 3.20$ & $14.65 \pm 4.76$ & $11.58 \pm 4.70$ & $11.19 \pm 4.10$ \\
\hline Thinking & $10.67 \pm 3.00$ & $10.22 \pm 3.55$ & $11.04 \pm 3.26$ & $11.04 \pm 3.00$ \\
\hline Upper extremity function* & $15.76 \pm 5.18$ & $18.39 \pm 5.27$ & $15.31 \pm 5.32$ & $16.42 \pm 5.43$ \\
\hline Vision & $12.69 \pm 2.68$ & $12.32 \pm 3.28$ & $12.09 \pm 2.98$ & $12.73 \pm 3.00$ \\
\hline Work/Productivity & $7.91 \pm 2.99$ & $8.15 \pm 4.20$ & $9.16 \pm 2.50$ & $9.89 \pm 2.98$ \\
\hline
\end{tabular}

All variables are mean \pm standard deviation (SD). CON: control group. ${ }^{*} \mathrm{p}<0.05$. SS-QOL: stroke specific quality of lifes

However, in the items of self-care, upper extremity function, the significant improvement results were shown. This is judged to be a result that was obtained because the mental practice that was carried out in this research was organized by being centered on the program of the training of the arms. Other than the research results ${ }^{3-5)}$ that the mental practice reported in the previous, many research results can improve the balance, the manual function, and the ability to carry out the everyday life of the stroke patients, such results mean that the mental practice can have the positive influences on their quality of life. But, as the limitations of this research, first, there is the point that there is a difficulty in generalizing the results as the number of the subjects is small and there is the point that the groups had the differences with regard to the factors that can influence the quality of life and that all such factors could not be controlled. As a result, it is thought that, in the future researches, the securing of more subjects and the analyses that control the diverse variables will be needed.

\section{REFERENCES}

1) Jackson PL, Lafleur MF, Malouin F, et al.: Potential role of mental practice using motor imagery in neurologic rehabilitation. Arch Phys Med Rehabil, 2001, 82: 1133-1141. [Medline] [CrossRef]

2) Choi JH, Choi YW, Nam KS, et al.: Effect of mental training on the balance control ability of healthy subject. J Phys Ther Sci, 2010, 22: 51-55. [CrossRef]

3) Dunsky A, Dickstein R, Marcovitz E, et al.: Home-based motor imagery training for gait rehabilitation of people with chronic poststroke hemiparesis. Arch Phys Med Rehabil, 2008, 89: 1580-1588. [Medline] [CrossRef]

4) Korn ER: The use of altered states of consciousness and imagery in physical and pain rehabilitation. J Ment Imagery, 1983, 7: 25-34.

5) Park J: Influence of mental practice on upper limb muscle activity and activities of daily living in chronic stroke patients. J Phys Ther Sci, 2016, 28: 1061-1063. [Medline] [CrossRef]

6) Jeon BJ: A case study on the SS-QOL of stroke patients receiving CIMT. J KSOT, 2004, 12: 39-48.

7) Paik YR, Kim SK: A task-oriented approach consisting of modified constraint-induced Movement Therapy(m-CIMT) and bimanual activity effects on upper extremity function and Activities of Daily Living (ADL) in stroke patients. J KSOT, 2010, 18: 79-94. 